\title{
Intra-operative recording of motor tract potentials at the cervico-medullary junction following scalp electrical and magnetic stimulation of the motor cortex
}

\author{
P D Thompson, B L Day, H A Crockard, I Calder, N M F Murray, J C Rothwell, \\ C D Marsden
}

\begin{abstract}
Activity in descending motor pathways after scalp electrical and magnetic brain stimulation of the motor cortex was recorded from the exposed cervicomedullary junction in six patients having trans-oral surgery of the upper cervical spine. Recordings during deep anaesthesia without muscle paralysis revealed an initial negative potential (D wave) at about $2 \mathrm{~ms}$ with electrical stimulation in five of the six patients. This was followed by a muscle potential which obscured any later waveforms. Magnetic stimulation produced clear potentials in only one patient. The earliest wave to magnetic stimulation during deep anaesthesia was 1-2 ms later than the earliest potential to electrical stimulation. Following lightening of the anaesthetic and the administration of muscle relaxants a series of later negative potentials (I waves) were more clearly seen to both electrical and magnetic stimulation. More I waves were recorded to magnetic stimulation during light anaesthesia than during deep anaesthesia. Increasing the intensity of electrical stimulation also produced an extra late I wave. At the highest intensity of magnetic stimulation the latency of the earliest potential was comparable to the D wave to electrical stimulation. The intervals between these various $D$ and $I$ waves corresponded to those previously described for the timing of single motor unit discharge after cortical stimulation.
\end{abstract}

A practical application of the techniques of electrical and magnetic stimulation of the motor cortex is intra-operative monitoring of motor tract function during spinal or cerebral surgery. The use of these techniques may lower the risk of intra-operative damage to motor tracts which on rare occasions complicate neurosurgical procedures without any warning from existing sensory evoked potential methods. ${ }^{1}$ Furthermore, abnormalities of motor conduction may be detected in the presence of normal sensory evoked potentials in some patients with spinal cord injury. ${ }^{2}$

There have been a number of studies of the motor tract potentials recorded from the thoracic and low cervical spinal cord after electrical brain stimulation. ${ }^{3-7}$ We had previously experienced difficulty in recording descending potentials to magnetic orain stimulation and the later waves to electrical brain stimulation at these lower spinal levels. We describe here the results recording the descending volleys produced by electrical and magnetic stimulation of the brain from the surface of the brainstem and high cervical cord. Access to these levels was provided by the surgical approach to the foramen magnum used by one of the authors (AC) so that recording electrodes could be placed on the anterior surface of the brain stem and high cervical spinal cord. ${ }^{8}$

Electrical stimulation

Deep anaesthesia: No muscle paralysis

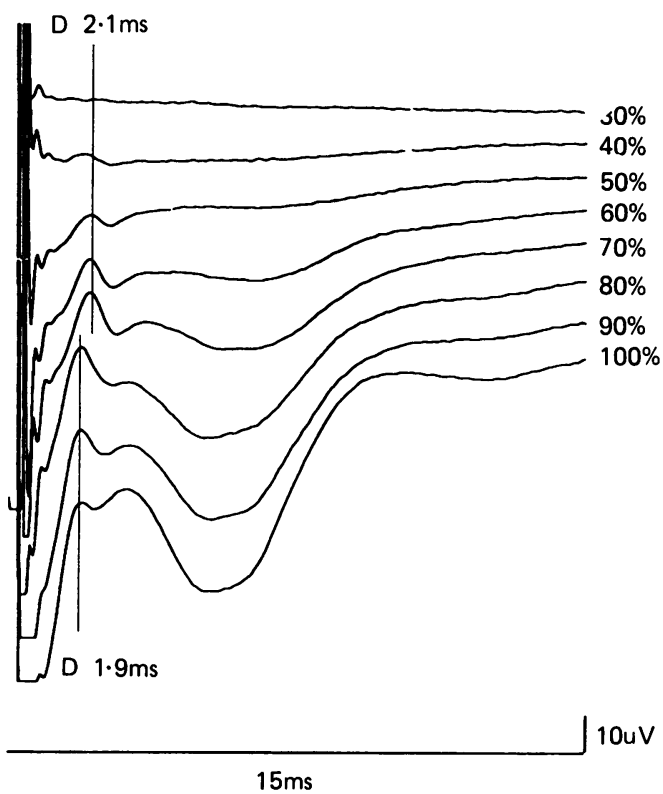

Figure 1 Cervico-medullary potentials recorded (in case 1) after electrical brain stimulation at increasing stimulus intensities. Stimuli were given at the start of the sweep. Each trace represents the average of 10 trials. At a stimulus intensity of $40 \%$ a negative potential at $2.1 \mathrm{~ms}$ is seen. This increases in size as the stimulus intensity is increased until it reaches a maximum at $70 \%$ and its latency shortens to $1.9 \mathrm{~ms}$. At higher intensities a later broad positive wave becomes apparent. This wave and the stimulus artefact intrude upon and tend to obscure the smaller earlier potential. The early wave represents descending activity in motor tracts produced by stimulation of the motor cortex. The larger and later positive wave represents muscle artefact (see figure 2). 
Electrical stimulation (70\%)

Deep anaesthesia: no muscle relaxants Light anaesthesia: muscle paralysis
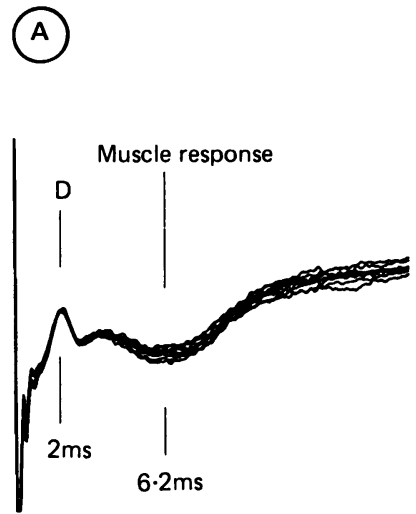

$\mathrm{J} 10 \mathrm{uV}$

$15 \mathrm{~ms}$
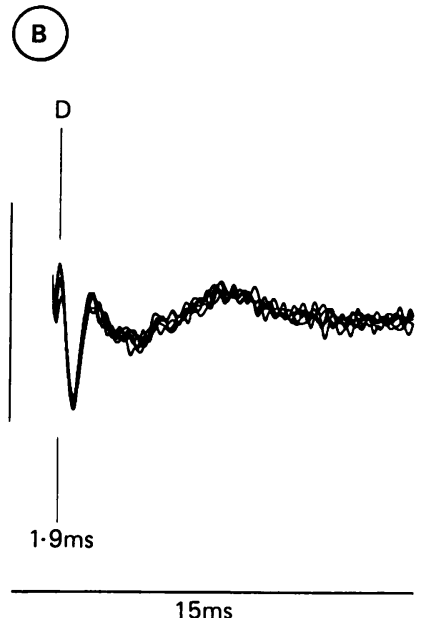

(C)

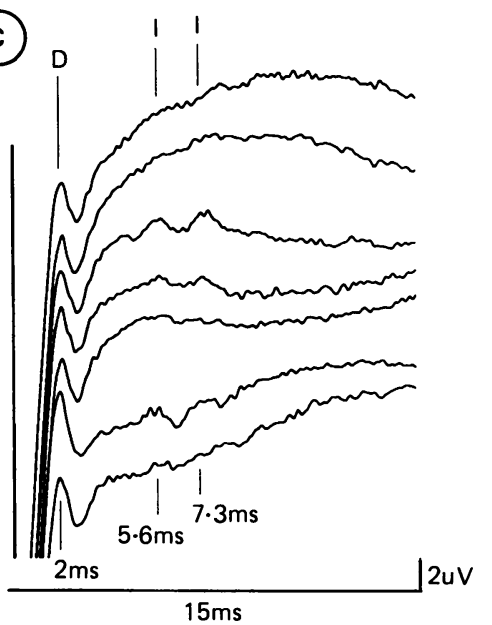

(D)

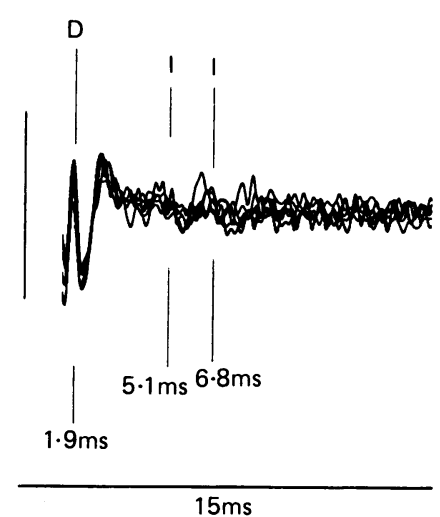

Figure 2 Cervico-medullary potentials recorded (in case 5) following electrical brain stimulation at $70 \%$ maximum. Stimuli were delivered at the start of the sweep. $A$ : During deep anaesthesia and without muscle paralysis. Single trials are superimposed. An early potential ( $D$ wave) at a latency of $2.0 \mathrm{~ms}$ is followed by later wave (positive peak latency $6.2 \mathrm{~ms}$ ). B: Differentiated trials shown in $A$ (superimposed). C: After the introduction of muscle paralysis and lightening of the anaesthesia 7 single trials are shown. The early $D$ wave is seen at $2 \mathrm{~ms}$ in all trials. The later positive potential is no longer evident following the introduction of muscle paralysis and lightening of the anaesthetic suggesting that it was a muscle response. Later I waves are evident on occasions, particularly trials 3 and 6.D:Differentiated traces from C. Again there is a suggestion of later waves on some trials although these are rather poorly defined. The stimulus artefact has been omitted from the differentiated traces.

\section{Patients and methods}

Seven patients (three females and four males) were studied. Their ages ranged from 34-79 years. Three patients had rheumatoid arthritis with high cervical spinal cord compression due to atlanto-axial arthropathy, three had congenital anomalies of the cranio-cervical junction, two of whom presented with myelopathy following trauma. One patient had an extensive chordoma. The clinical details are summarised in the table.

All patients were premedicated with papaveretum and hyoscine then anaesthetised with thiopentone or propofol and intubated with a fibreoptic laryngoscope. The effect of anaesthesia and muscle paralysis on the potentials recorded following stimulation of the motor cortex was studied in four patients who breathed halothane or enflurane in nitrous oxide and oxygen during transoral odontoidectomy and were then paralysed with atracurium and ventilated with $30 \%$ oxygen in nitrous oxide. Additional analgesics were given at this time. Ventilation was continued for about 20-35 minutes until no volatile agent was detected in the circuit by the volatile agent monitor (Datex Normac). Two patients were ventilated throughout the procedure and volatile agents were discontinued as outlined above. One patient breathed spontaneously throughout the procedure.

The surgical approach to these lesions was through the mouth to the posterior pharynx. The patients were anaesthetised, paralysed, intubated and ventilated. The arch of $\mathrm{C} 1$ was exposed by a midline incision through the posterior pharynx. The arch of $\mathrm{Cl}$ and the odontoid peg were then removed exposing the dura in front of the foramen magnum. At this point the surgeon inserted the recording electrodes (USC1-4F cardiac pacing electrodes: inter-electrode distance $1 \mathrm{~cm}$ ) into the exposed epidural space. They were placed on the surface of the dura overlying the cervicomedullary junction in six of the seven patients, and adjacent to the ponto-medullary region in the patient with a clivus chordoma.

Electrical brain stimulation was performed using a Digitimer D-180 stimulator via two $\mathrm{Ag} / \mathrm{AgCl}$ surface electrodes $(9 \mathrm{~mm}$ in diameter) fixed to the scalp with collodion. A large interelectrode distance was chosen to maximise the size of the descending potentials by placing the anode and cathode over each hand area of the scalp (at a point $7 \mathrm{~cm}$ lateral to the vertex). The hand areas were chosen since the recording electrodes were positioned over the descending spinal tracts destined to innervate the motor neurons supplying muscles of the hand and arms. A variety of

Table Summary of clinical details, examination findings and central motor conduction times (CMCT) for abductor digiti minimi in the upper limbs and tibialis anterior in the lower limbs in the seven patients described in this report. Intraoperative recordings were made from the cervico-medullary junction in cases 1, 3-7 and from the medullary pyramids in case 2

\begin{tabular}{|c|c|c|c|c|c|c|c|c|c|c|c|}
\hline \multirow[b]{2}{*}{ Case } & \multirow[b]{2}{*}{ Age } & \multirow[b]{2}{*}{ Sex } & \multirow[b]{2}{*}{ Diagnosis } & \multicolumn{2}{|c|}{ UMN Signs } & \multicolumn{2}{|c|}{ Sensory Signs } & \multirow[b]{2}{*}{$R U L$} & \multicolumn{3}{|c|}{ Central Motor Conduction } \\
\hline & & & & $U L$ & $L L$ & $U L$ & $L L$ & & $L U L$ & $R L L$ & $L L L$ \\
\hline $\begin{array}{l}1 \\
2 \\
3 \\
4 \\
5 \\
6 \\
7\end{array}$ & $\begin{array}{l}60 \\
35 \\
71 \\
43 \\
36 \\
56 \\
59\end{array}$ & $\begin{array}{l}\mathrm{F} \\
\mathrm{M} \\
\mathrm{M} \\
\mathrm{M} \\
\mathrm{F} \\
\mathrm{M} \\
\mathrm{F}\end{array}$ & $\begin{array}{l}\text { Rheumatoid Arthritis } \\
\text { Clivus Chordoma } \\
\text { Congenital/Traumatic } \\
\text { Congenital/Traumatic } \\
\text { Platybasia/Syringomyelia } \\
\text { Rheumatoid Arthritis } \\
\text { Rheumatoid Arthritis }\end{array}$ & $\begin{array}{l}+ \\
+ \\
+ \\
+ \\
+ \\
+ \\
+\end{array}$ & $\begin{array}{l}- \\
+ \\
+ \\
+ \\
+ \\
+ \\
+\end{array}$ & $\begin{array}{l}+ \\
- \\
- \\
+ \\
+ \\
+ \\
+\end{array}$ & $\begin{array}{l}- \\
- \\
\overline{+} \\
+ \\
+\end{array}$ & $\begin{array}{l}\mathrm{N} \\
\mathrm{N} \\
\text { Abn }(12 \cdot 7) \\
\mathrm{N} \\
\text { Abn }(8 \cdot 8) \\
\text { Abn }(8 \cdot 5) \\
\mathrm{N}\end{array}$ & $\begin{array}{l}\mathrm{N} \\
\mathrm{N} \\
\text { Abn }(14 \cdot 6) \\
\mathrm{N} \\
\text { Abn }(9 \cdot 6) \\
\text { Abn }(8 \cdot 4) \\
\text { ND }\end{array}$ & $\begin{array}{l}\mathrm{N} \\
\text { ND } \\
\text { Absent } \\
\text { ND } \\
\text { Absent } \\
\text { Abn }(32 \cdot 1) \\
\text { ND }\end{array}$ & $\begin{array}{l}\mathrm{N} \\
\text { ND } \\
\text { ND } \\
\text { ND } \\
\text { Absent } \\
\text { ND } \\
\text { ND }\end{array}$ \\
\hline
\end{tabular}

Footnotes: $\mathrm{UMN}=$ upper motor neurone syndrome. $\mathrm{UL}=$ upper limb, $\mathrm{LL}=$ lower limb, $\mathrm{R}=$ right, $\mathrm{L}=$ left. $\mathrm{N}=$ normal, Abn $=$ abnormal $(\mathrm{value}(\mathrm{ms})$ ), Footnotes: $\mathrm{UMN}=$ upper motor neurone syndrome. UL $=$ upper limb, $\mathrm{LL}=$ lower limb, $\mathrm{R}=$ right, $\mathrm{L}=\mathrm{left} . \mathrm{N}=\mathrm{normal}, \mathrm{Abn}=\mathrm{abnormal}(\mathrm{value}(\mathrm{ms}))$,
$\mathrm{ND}=$ not done. $\mathrm{C} 2=$ sensory impairment in upper cervical dermatomes. Normal CMCT values for abductor digiti minimi: mean $(\mathrm{SD}) 6 \cdot 2(0 \cdot 86) \mathrm{ms}$ : range $4 \cdot 5-$ $\mathrm{ND}=$ not done. $\mathrm{C} 2$ = sensory impairment in upper cervical dermatomes. Normal CMCT values
$7.7 \mathrm{~ms}(9)$. Normal absolute latency for tibialis anterior: mean (SD) $29 \cdot 3$ (1) ms; range 27.3-31.3 ms. 
Electrical stimulation (90\%)
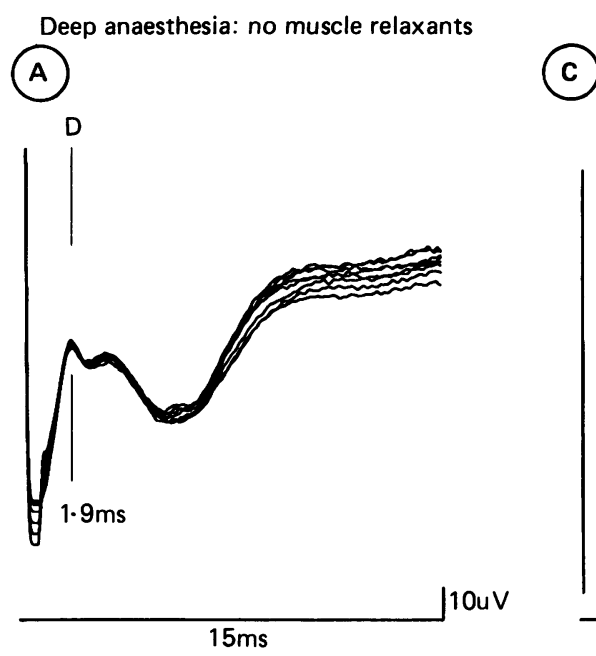

Light anạesthesia: muscle paralysis

C)

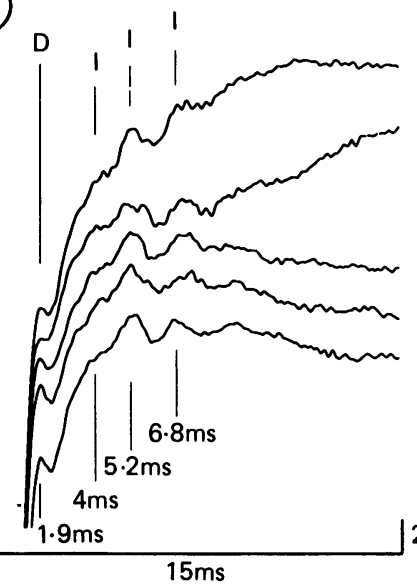

(B)

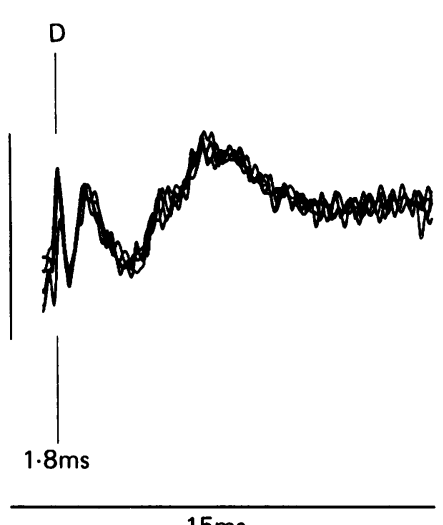

$15 \mathrm{~ms}$
(D)

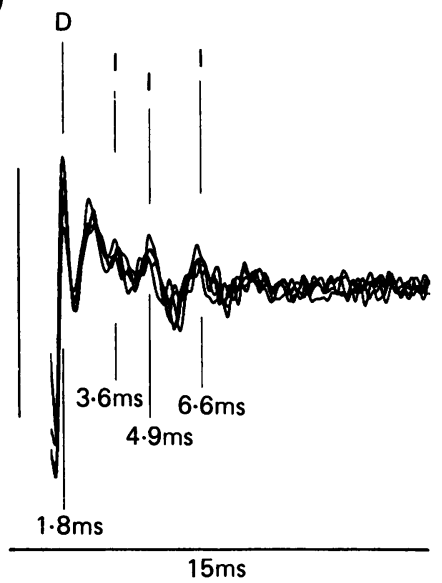

Figure 3 Cervico-medullary potentials as for figure 2 (case 5) to electrical stimulation at intensity of $90 \%$. A: Deep anaesthesia, no muscle relaxants. The early negative potential is again evident and is followed by a large positive wave. B: Differential of traces shown in $A$. Small waves can now be seen superimposed on the broad later wave. $C:$ Following lightening of the anaesthetic and the introduction of muscle paralysis the later waves are clearly seen and are larger than those in figure 2. An additional earlier wave also has been recruited at $4 \mathrm{~ms}$. D: Differential of records shown in $C$ where the later waves are more clearly seen. Note the small shift in the peak latency of these waves in comparison with the raw traces in $C$. This is because the peak of the differentiated raw waveforms corresponds to the maximal slope of the raw waves and therefore occurs a fraction of a millisecond earlier than their peak latency. The stimulus artefact has been omitted from the differentiated traces.

stimulus intensities was used, from $10-100 \%$ of the maximal output of the stimulator. The maximal output is 700 volts with a peak current of about $1 \mathrm{~A}$. Magnetic stimulation was performed using a Novametrix Magstim $200(2 \cdot 1 \mathrm{~T}$, peak at $150 \mu$ s at maximum output of the device). The coil ( $9 \mathrm{~cm}$ in diameter) was centred on the vertex ("A-side" up) and a range of stimulus intensities from 20 to $100 \%$ of the maximum output were used. Brain stimulation was performed before and after the change in anaesthesia in five patients. A series of trials (5-20) were collected at each intensity. The signals were amplified using Digitimer D150 amplifiers (filter settings $1.6 \mathrm{~Hz}-3 \mathrm{kHz}$ ). The responses were recorded on tape (RACAL 7DS) for later playback. Raw signals were also differentiated digitally (consecutive differences) and then smoothed with a three point moving averaging filter using computer software. This has the effect of

removing slower potentials including the deflection created by amplifier recovery following the stimulus and accentuating the descending volleys which have a higher frequency but smaller amplitude.

All patients had pre-operative magnetic brain stimulation studies to determine central motor conduction times (CMCT). ${ }^{9}$ Magnetic brain stimulation was performed as described above, but with the subject making a modest voluntary contraction of the abductor digiti minimi (ADM) muscle. In four cases (cases 14) percutaneous electrical stimulation over the lower cervical spine was used to excite the C8 and $\mathrm{T} 1$ spinal roots at the intervertebral foramina, and the latency to cervical stimulation was subtracted from that to brain stimulation to obtain the CMCT. In three subjects cervical stimulation was not employed on account of the patients' cervical spine disease: in two (cases 5,6 ) of these CMCT was measured indirectly, using $F$ wave latencies to wrist stimulation to measure the peripheral component, and in one patient (case 7) only absolute latencies to brain stimulation were measured. Conduction to the lower limbs was assessed in four patients using recording electrodes on tibialis anterior muscles.

\section{Results}

Pre-operative Studies

Pre-operative central motor conduction times (CMCT) or measurements of the latency to onset of muscle action potentials in upper and lower limb muscles were normal in three patients and the absolute latency from cortical stimulation was normal in one. CMCTs were moderately prolonged bilaterally in one subject $(12.7$ and $14.6 \mathrm{~ms})$, and slightly prolonged in two. Latency of responses from the tibialis anterior were normal in one subject (with normal upper limb responses), slightly delayed in one, as were the upper limb responses and unobtainable in two. The clinical findings and CMCT measurements are summarised in the table.

\section{Intra-operative Studies}

Recordings from the cervico-medullary junction Electrical motor cortex stimulation

In the deeply anaesthetised and unparalysed state, a short latency (range of peak latencies $1 \cdot 9-2 \cdot 1 \mathrm{~ms})$ negative potential was seen with electrical stimulation in five of the six patients. The remaining patient (case 3 ) was shown preoperatively to have abnormal central motor conduction. The estimates of CMCT were slightly delayed in cases 5 and 6 but the latency of the first peak recorded from the cervicomedullary junction in these patients fell within the above range. The early negative potential was first evident at stimulus intensities of about $40 \%$ (fig 1). It increased in size and shortened in latency (by $0 \cdot 1-0.2 \mathrm{~ms}$ ) with increasing intensities of stimulation. There was little change in the amplitude of the potential above stimulus intensities of the order of $60-70 \%$. Stimulus artefact often obscured this early wave at 
Magnetic stimulation (80\%)

Deep anaesthesia: No muscle relaxants Light anaesthesia: Muscle paralysis

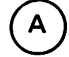

(c)
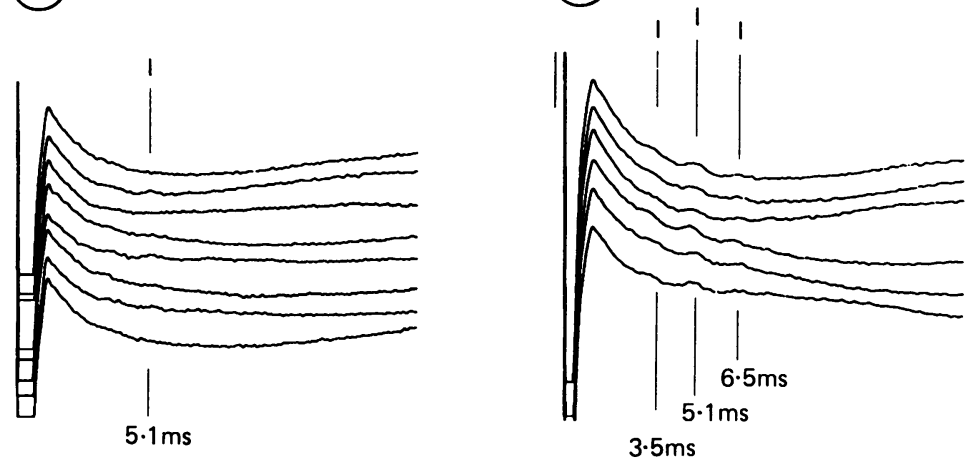

$5 u V$

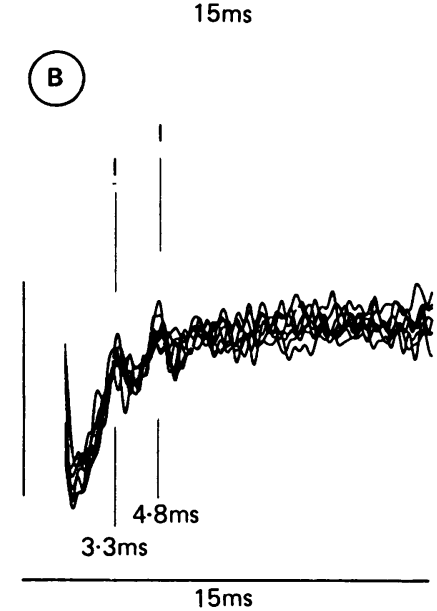
$」 5 \mathrm{uV}$

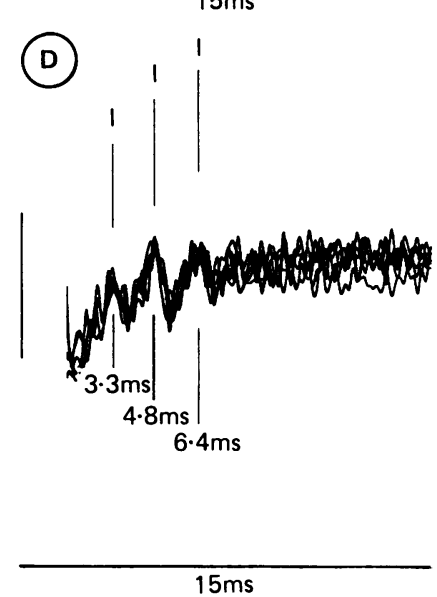

Figure 4 Cervico-medullary potentials as for figures 2 and 3 (case 5) following magnetic stimulation at intensity $80 \%$. A : During deep anaesthesia and without muscle relaxants a small inflection can just be discerned at $5 \cdot 1 \mathrm{~ms}$. No early potential is seen and a muscle response is not present. B. The differential of the traces shown in $A$ reveals 2 waves, but no early potential is present corresponding to the earliest wave to electrical stimulation. $C$ : Light anaesthesia and muscle paralysis. Three waves are now visible in the raw record. These are more clearly seen in the differentiated traces shown in panel D: Stimulus artefact omitted from differentiated traces.

higher intensities. The early negative wave was followed by a larger positive potential (peak positive latency 4-9ms; duration 5-10 ms).

Following lightening of the anaesthetic and introduction of muscle paralysis the large positive wave disappeared, suggesting that it was a muscle response (compare fig $2 \mathrm{~A}$ and $\mathrm{C}$ ). The early negative potential was again evident. In two patients (cases 5 and 6) later negative waves were recorded. The responses recorded in case 5 are illustrated in fig 2 . Unlike the earliest wave which was present on all trials, and easily seen on a single sweep, the later waves were more variable and best seen after inspection of several trials or differentiating the raw traces (fig $2 \mathrm{D}$ ). At $70 \%$ intensity two later waves were recorded at latencies of about 5 and $6-7 \mathrm{~ms}$. Increasing the stimulus intensity from $70 \%$ to $90 \%$ recruited these waves more consistently and also an extra wave at a latency of about 3-4ms (fig 3). Note that in these and subsequent illustrations the latency to the peak of the potentials in the differentiated traces inevitably is a fraction of a millisecond shorter than the peaks in the raw data. This effect can be ascribed to the differentiation process producing a peak at the maximum slope of the raw potentials.

\section{Magnetic stimulation}

Muscle contraction was less of a problem with magnetic stimulation but the stimulus artefact was large. During deep anaesthesia later potentials were seen in only one patient (case 5, who also exhibited multiple waves to electrical stimulation) (fig 4). These waves were small and their appearance varied from trial to trial. At stimulus intensity $80 \%$ two waves at latencies of 3.3 and $4.8 \mathrm{~ms}$ are clearly seen on the differentiated trace, while under deep anaesthesia (fig 4B). Following removal of the volatile anaesthetic and the introduction of muscle paralysis, these waves were again evident in only this patient but an extra later potential at $6.4 \mathrm{~ms}$ was also seen. These waves were largest in size at the highest intensities of stimulation and during light anaesthesia (fig 5). The later wave at about $6.5 \mathrm{~ms}$ was recorded at intensities of 80 and $100 \%$ during light anaesthesia, but was not seen at intensities of 80 or $90 \%$ during deep anaesthesia. This suggests that its appearance may have been related to the change in anaesthesia. The early potential seen with electrical stimulation at between 1.9 and $2.1 \mathrm{~ms}$ was not seen in the records at magnetic stimulus intensities of 80 and $90 \%$. However, an early potential at $2 \mathrm{~ms}$, comparable in latency to the early potentials recorded to electrical stimulation can be seen following the stimulus artefact in the differentiated records (fig 5D).

\section{Recordings from the medullary pyramids}

In the one patient in whom the operative site extended as high as the pontomedullary junction, stimulus artefact from both devices was large and it was not possible to discern any descending potentials.

\section{Discussion}

These results extend the findings of previous studies demonstrating that potentials may be recorded from the descending motor tracts in response to electrical stimulation of the brain during spinal surgery. The recordings described here were made from the highest levels of the spinal cord at the cervico-medullary junction. Stimulus artefact posed considerable difficulties when recording descending activity from the cervico-medullary junction particularly following magnetic stimulation, and it proved impossible to record from the medullary pyramid with electrical or magnetic stimuli. Although the presence of significant cervical cord compression in these cases may also have contributed to the failure to record potentials in some subjects (all but one had signs of an upper motor neuron syndrome in their upper and lower limbs), preoperative studies were normal in four. The one patient (case 5) in whom it was possible to make a detailed comparison of the potentials following electrical and magnetic stimulation had slightly prolonged preoperative estimates of central motor conduction times. The latencies of the initial descending volleys in this case were comparable to those recorded in the other cases, suggesting that the site of any compromise of spinal function was below the level at which the recordings were made. 


\section{Magnetic stimulation}

Deep anaesthesia: no muscle relaxants
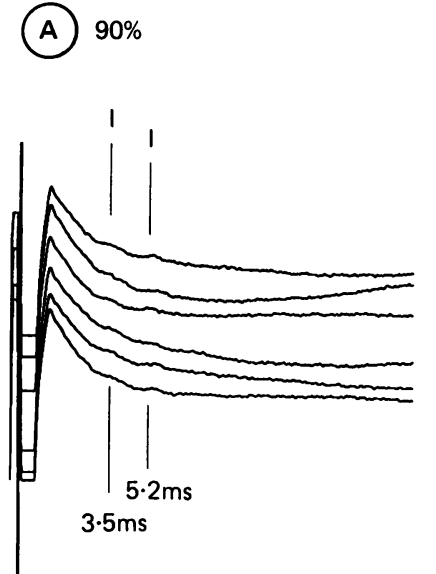

$」 5 u V$

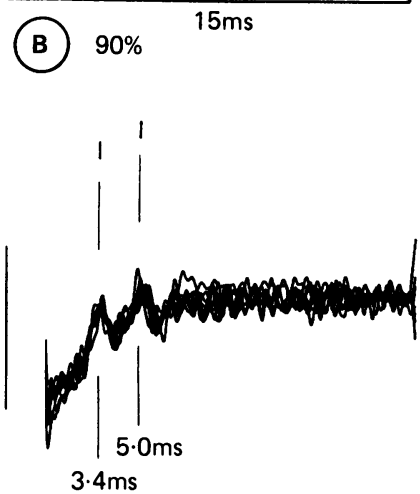

$15 \mathrm{~ms}$
Light anaesthesia: muscle paralysis (C) $100 \%$

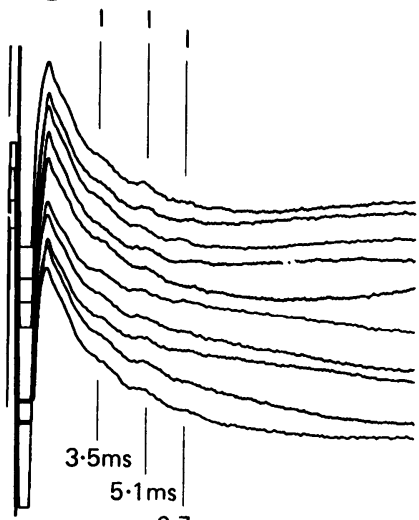

$6 \cdot 7 \mathrm{~ms}$
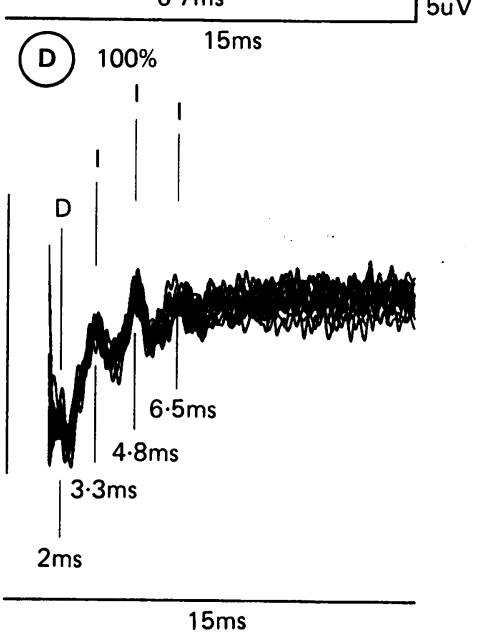

Figure 5 Cervico-medullary potentials as for previous figures (case 5) following magnetic stimulation at $90 \%$ during deep anaesthesia and without muscle relaxants (panels $A$ and $B$ ) and at $100 \%$ following lightening of the anaesthetic and introduction of muscle relaxants. $A$ : Two waves are just visible. $B$ : These are more clearly shown in the differentiated records. C: At the higher stimulus intensity following lightening of the anaesthetic and introduction of muscle paralysis, three waves are evident. D: These waves are more clearly seen in the differentiated traces. Note that the effect of altering the anaesthetic is the same as for intensity $80 \%$ with the appearance of an extra wave at about $6.5 \mathrm{~ms}$, suggesting that the recruitment of this wave was related to the anaesthetic change. An extra earlier wave also is evident in the differentiated traces at stimulus intensity $100 \%$. The latency of this wave corresponds to that of the earliest potential to electrical stimulation and was only seen at this highest intensity of magnetic stimulation. Stimulus artefact omitted from differentiated traces.

Electrical stimulation elicited a response recorded from the cervico-medullary junction in five of six patients at a latency of about $2 \mathrm{~ms}$. In the other patient we could not record a response but this may have been due to considerably delayed central motor conduction and dispersion of the descending motor volley. After lightening of the anaesthetic and the administration of muscle relaxants later waves recorded from the cervico-medullary junction were clearly seen in two of the five patients at latencies of 3-4, 5 and $6-7 \mathrm{~ms}$. The earliest of these potentials was recruited only at the highest stimulus intensity. Magnetic stimulation in one of these patients also produced waves that roughly corresponded in time to the later waves following electrical stimulation at latencies of $3-4,5$ and $6-7 \mathrm{~ms}$. With magnetic stimulation the wave at $6-7 \mathrm{~ms}$ was seen only after the change in anaesthesia with intensities of 80 and $100 \%$, but was not evident during deep anaes- thesia with intensities of 80 or $90 \%$. At the highest intensity of magnetic stimulation $(100 \%)$ an early potential at $2 \mathrm{~ms}$, corresponding to the earliest potential to electrical stimulation, was seen in the differentiated records.

The potentials produced by electrical stimulation (in contrast to magnetic stimulation) included a later potential which was abolished by muscle paralysis. This was probably a muscle response caused by direct stimulation of peripheral nerves innervating cranial or paraspinal muscles. In our experience, such muscle twitches are commonly seen in awake intact subjects during electrical stimulation over the scalp, but are less conspicuous with magnetic stimulation with the coil centred over the vertex. In the patients studied here, facial twitches were clearly seen following electrical stimulation before muscles were paralysed, but were much reduced after paralysis.

\section{Physiological considerations}

We have previously suggested on the basis of our EMG studies that electrical stimulation of the motor cortex through the scalp in awake intact humans produces multiple descending volleys (at intervals of 1.5 to $2 \mathrm{~ms}$ ) in the pyramidal tract. ${ }^{10-12}$ Our hypothesis is that the first of these volleys to electrical stimulation is caused by direct activation of corticospinal axons (the $\mathrm{D}$ wave), while the later volleys are due to indirect or synaptic activation of the same corticospinal neurons (I waves). Our previous estimate of the central conduction time of the $D$ wave to the $C 5$ level of the spinal cord was of the order of $4 \mathrm{~ms}$. Accordingly, the earliest potential (at latencies of $1 \cdot 9-2 \cdot 1 \mathrm{~ms}$ ) recorded from the cervico-medullary region following electrical stimulation corresponds to the $\mathrm{D}$ wave. The intervals at which the later waves followed the initial (D) wave to electrical stimulation in the one detailed study described here were about 1-2 ms, $3 \mathrm{~ms}$ and $4-5 \mathrm{~ms}$. These intervals correspond closely to the intervals between the peaks of probability of discharge of single motor units following electrical cortical stimulation in intact humans. These peaks of increased firing probability occurred at $1-2 \mathrm{~ms}, 2.5-3.5 \mathrm{~ms}$ and $4-5.5 \mathrm{~ms}$ following the first peak. ${ }^{12}$ It seems plausible therefore that the later waves sometimes seen with electrical stimulation in this study are the same as those deduced from EMG studies in intact humans. According to our hypothesis, these later waves would represent indirect (I) activation of corticospinal neurons. The timing of these waves and their recruitment order with electrical stimulation are also similar to the I waves in the baboon where the earliest I wave was only recorded at the highest intensities of electrical stimulation. ${ }^{13}$

With magnetic stimulation there are differences in the latency of EMG responses which, we have argued, can be explained by predominantly indirect activation of corticospinal neurons. ${ }^{112}$ Our results agree with this hypothesis. Following magnetic stimulation, an early potential was identified only at the highest intensity of stimulation. Although D 
waves are most effectively produced by electrical stimulation there is some evidence that they may also be elicited by magnetic stimulation in certain circumstances. ${ }^{1214-16}$ The earliest potential recorded to magnetic stimulation in the present study at stimulus intensity $100 \%$ may represent such a direct potential. The latency of the remaining waves to magnetic stimulation was comparable to that of the later waves with electrical stimulation and we suggest these represent I waves. The last of these waves was seen only after lightening of the anaesthetic suggesting that they might be susceptible to the level of anaesthesia. Although the mechanism of action of volative anaesthetics is poorly understood, some of their effects are thought to be mediated by interference with synaptic transmission. For example, cortical somatosensory evoked potentials are attenuated by volatile anaesthetics. ${ }^{1}$ Such interference with the synaptic activation of pyramidal neurons by brain stimulation might also contribute to the difficulties in recording later waves. Direct activation of corticospinal axons as seen with electrical stimulation, however, remains virtually unaltered. In the case of electrical stimulation, the removal of muscle artefact alone might be sufficient to account for the appearance of later waves. Indeed, the differentiated traces in figure 3B suggest that there are small later I waves superimposed on the larger muscle response. With electrical stimulation, the effect of increasing stimulus intensity was to recruit an earlier wave at a latency of $3-4 \mathrm{~ms}$. The latency of this wave corresponded to that of the first wave recorded to magnetic stimulation (at just suprathreshold intensities).

Shortening of $\mathrm{D}$-wave latency with increasing intensities of stimulation has been previously described in animal experiments (cat, ${ }^{17}$ monkey ${ }^{1415}$ ) and studies in humans. ${ }^{1418}$ The reduction in D-wave latency can be up to $1.7 \mathrm{~ms}$ in humans ${ }^{18}$ suggesting excitation of the pyramidal tracts at progressively deeper levels within the brain with increasing intensities of stimulation. This occurs in a stepwise manner consistent with preferential activation at different sites along the pyramidal tract. The extent of latency shortening in the present cases (of the order of $0.2 \mathrm{~ms}$ ) was smaller than that seen in the study of Burke et al. ${ }^{18}$ This may be explained by the difficulties in recording from the cervico-medullary junction at the highest stimulus intensities. We were unable to make recordings at the highest intensities of stimulation in these patients at this level of the spinal cord and therefore may not have reached the intensities where the maximal latency shortening occurs. The technical difficulties encountered when recording at these levels may also explain the smaller size of the $\mathrm{D}$-wave potentials in this study (figs 2 and 3 ).

Finally, these results have some practical implications. For monitoring purposes, examination of the $\mathrm{D}$ wave elicited by electrical stimulation appears to be the most practical method and has several advantages over magnetic stimulation. It is simpler to perform since the stimulating electrodes are much smaller than the stimulating coil and may be fixed in place preoperatively. Furthermore, focal activation of a particular region of the motor cortex is much easier with electrical stimulation, where it is simply a matter of electrode placement; magnetic stimulation tends to be more diffuse, unless specially designed coils are used or there is voluntary activation of the desired muscle group. The scalp discomfort which is a major disadvantage of electrical stimulation of the conscious subject is of course not a problem during surgery with anaesthesia and analgesia. Recording from the spinal cord circumvents any difficulties recording surface EMG responses during neuromuscular blockade, but introduces other technical problems because of stimulus artefact at these high levels of the spinal cord.

1 Lesser RP, Raudzens P, Luders $\mathrm{H}$, et al. Post operative neurological deficits may occur despite unchanged intraoperative somatosensory evoked potentials. Ann Neurol 1986;19:22-5.

2 Thompson PD, Dick JPR, Asselman P, et al. Examination of motor function in lesions of the spinal cord by stimulation of the motor cortex. Ann Neurol 1987;21:389-96.

3 Levy WJ, York DH. Evoked potentials from the motor tracts in man. Neurosurg 1983;12:422-9.

4 Boyd S, Rothwell JC, Cowan JMA, et al. A method of monitoring function in corticospinal pathways during scoliosis surgery with a note on motor conduction velocities. J Neurol Neurosurg Psychiatry 1986;49:251-7.

5 Pelosi L, Caruso G, Balbi P. Characteristics of spinal potentials to transcranial motor cortex stimulation: potentials to transcranial motor cortex stimulation:
Intraoperative recording. In: Rossini $P$, Marsden CD, eds. Non-invasive stimulation of brain and spinal cord. New York: Alan R Liss 1988:297-304

6 Katayama Y, Tsobokawa T, Maejima S, Hirayama T, Yamamoto $\mathrm{T}$. Corticospinal direct response in humans: identification of the motor cortex during intracranial surgery under general anaesthesia. $J$ Neurol Neurosurg Psychiatry 1988;51:50-9.

7 Inghilleri M, Berardelli A, Cruccu G, Priori A, Manfredi $M$. Corticospinal potentials after transcranial stimulation in intact man. J Neurol Neurosurg Psychiatry 1989;52:970-4.

8 Crockard HA. Anterior approaches to lesions of the upper cervical spine. Clin Neurosurg 1988;34:389-416.

9 Hess CW, Mills KR, Murray NMF, Schreifer TN. Magnetic brain stimulation in multiple sclerosis. Ann Neurol 1987;22:744-52.

10 Day BL, Rothwell JC, Thompson PD, et al. Motor cortex stimulation in man. 2. Multiple descending volleys. Brain 1987;110:1191-210

11 Day BL, Thompson PD, Dick JPR, Nakashima K, Marsden $\mathrm{CD}$. Different sites of action of electrical and magnetic stimulation of the human brain. Neurosci Lett 1987; 75:101-6.

12 Day BL, Dressler D, Maertens de Noordhout A, et al. Electric and magnetic stimulation of the human motor cortex: surface EMG and single motor unit responses. $J$ Physiol (Lond) 1989;412:449-73.

13 Kernell D, Wu CP. Responses of the pyramidal tract to stimulation of the baboon's motor cortex. J Physiol (Lond) 1967;191:653-72.

14 Amassian VE, Quirk GJ, Stewart $M$ A comparison of corticospinal activation by magnetic coil and electrical stimulation of monkey cortex. Electroencep Clin Neurostimulation of monkey

15 Edgley SA, Eyre JA, Lemon RN, Miller JSG. Excitation of the corticospinal tract by electro-magnetic and electrical stimulation of the scalp in the macacque monkey. J Physio Lond) 1990;425:301-20.

16 Berardelli A, Inghilleri M, Cruccu G, Manfredi M. Descending volley after transcranial electrical and magnetic stimulation in man. Neurosci Lett 1990;112:54-8.

17 Landau WM, Bishop GH, Clare MH. Site of excitation in stimulation of the motor cortex. J Neurophysiol 1965; 28:1206-22.

18 Burke D, Hicks RG, Stephen PH. Corticospinal volleys evoked by anodal and cathodal stimulation to the human motor cortex. J Physiol (Lond) 1990;425:283-99. 